\title{
Aspiration Level Methods in Interactive Multi-objective Programming and Their Engineering Applications (Abstract of Invited Talk)
}

\author{
Hirotaka Nakayama \\ Konan University \\ 8-9-1, Okamoto, Higashinada-ku, Kobe Hyogo, 658-8501, Japan \\ nakayama@konan-u.ac.jp
}

\begin{abstract}
One of the most important tasks in multi-objective optimization is "trade-off analysis" which aims to make the total balance among objective functions. The trade-off relation among alternatives can be shown as Pareto frontier. In cases with two or three objective functions, the set of Pareto optimal solutions in the objective function space (i.e., Pareto frontier) can be depicted relatively easily. Seeing Pareto frontiers, we can grasp the trade-off relation among objectives totally. Therefore, it would be the best way to depict Pareto frontiers in cases with two or three objectives. (It might be difficult to read the trade-off relation among objectives with three dimension, though). In cases with more than three objectives, however, it is impossible to depict Pareto frontier. There are some cases with a large number (e.g., a few hundreds) of objective functions in engineering applications such as erection management of cable stayed bridges and optical lens design. Under this circumstance, interactive methods can help decision makers (DMs) to make local trade-off analysis through interaction of DMs and computers by showing a Pareto solution nearest to their desire. Along this line, aspiration level methods were developed, and have been observed to be effective in many practical problems in various fields. Satisficing Trade-off Method proposed by the author is one of aspiration level methods, and has several devices for making trade-off analysis easily, i.e., automatic trade-off and exact trade-off. This paper discusses those methods for multi-objective optimization, in particular, from a viewpoint of engineering application.
\end{abstract}

